Supplementary material

\title{
Probing Angle-Dependent Interlayer Coupling in Twisted Bilayer $\mathrm{WS}_{2}$
}

Wei Yan, ${ }^{1 \dagger}$ Lan Meng, ${ }^{2 \dagger}$ Zhaoshun Meng, ${ }^{1}$ Yakui Weng, ${ }^{1}$ Lulu Kang, ${ }^{1}$ Xing-ao Li, ${ }^{1 *}$

${ }^{1}$ New Energy Technology Engineering Laboratory of Jiangsu Province \& School of Science, Nanjing University of Posts and Telecommunications (NUPT), Nanjing 210023, China

${ }^{2}$ College of Electronic and Optical Engineering \& College of Microelectronics, Nanjing University of Posts and Telecommunications, Nanjing 210023, China

Methods:

CVD synthesis of monolayer $\mathrm{WS}_{2}$ film

The monolayer $\mathrm{WS}_{2}$ film were grown on silicon substrates with a $300 \mathrm{~nm}$ layer of $\mathrm{SiO}_{2}$ by CVD method at atmospheric pressure with $\mathrm{S}$ and $\mathrm{WO}_{3}$ as precursors. $\mathrm{S}$ powder (Alpha Aesar, 99.5\%, $300 \mathrm{mg}$ ) and $\mathrm{WO}_{3}$ powder (Alpha Aesar, 99.5\%, 30 mg) were placed in a horizontal tube with two furnaces (low temperature zone and high temperature zone) respectively. $\mathrm{SiO}_{2} / \mathrm{Si}$ substrates were placed in growth zone which were $2 \mathrm{~cm}$ after the alumina crucible with $\mathrm{WO}_{3}$. Before heating, the tube was pumped down to $20 \mathrm{~Pa}$ and flushed with 300 sccm high-purity Ar gas several times to exclude oxygen, followed by filling Ar gas until the pressure in the tube is atmospheric pressure. Subsequently, Ar gas with flow rate of 120 sccm was introduced and kept until to the end of the experiment. During the heating process, 
the low temperature zone for S precursors was first kept at room temperature for 20 minutes, then heated to $250^{\circ} \mathrm{C}$ in 8 minutes, and held for 7 minutes. Meanwhile, the high temperature furnace for $\mathrm{WO}_{3}$ precursors and $\mathrm{SiO}_{2} / \mathrm{Si}$ substrates was heated from room temperature to $1150^{\circ} \mathrm{C}$ and $880^{\circ} \mathrm{C}$ in 35 minutes respectively, then held for 15 minutes for growth. After growth process, the furnace was removed to allow the substrate cooled naturally. Schematic growth process is shown in Figure S1(a).

Fabrication of twisted bilayer $\mathrm{WS}_{2}$

Twisted bilayer $\mathrm{WS}_{2}$ were fabricated using a PMMA stamping method. The top layer grown on $\mathrm{SiO}_{2} / \mathrm{Si}$ was spin coated with PMMA to protect the monolayer $\mathrm{WS}_{2}$. After spin coating, the sample was annealed at $100^{\circ}$ for 10 minutes. Then the $\mathrm{PMMA} \mathrm{WS}_{2}$ sample were etched using 20\% potassium hydroxide (KOH). Subsequently, the PMMA/ $\mathrm{WS}_{2}$ was rinsed in DI water to reduce reagent. The PMMA/WS $S_{2}$ monolayer was stamped onto a $\mathrm{SiO}_{2} / \mathrm{Si}$ substrate grown with $\mathrm{WS}_{2}$ monolayers in advance. Afterwards, PMMA was removed in acetone steam. After removing of PMMA, vertically stacked bilayer $\mathrm{WS}_{2}$ with various twisted angle were formed on $\mathrm{SiO}_{2} / \mathrm{Si}$ substrate. Finally, the as-prepared bilayer samples were thermal annealed under protective atmosphere at $300^{\circ}$ for $3 \mathrm{~h}$ to further clean the sample 
and enhance the interlayer coupling between two $\mathrm{WS}_{2}$ monolayers. Schematic transfer process is shown in Figure S2.
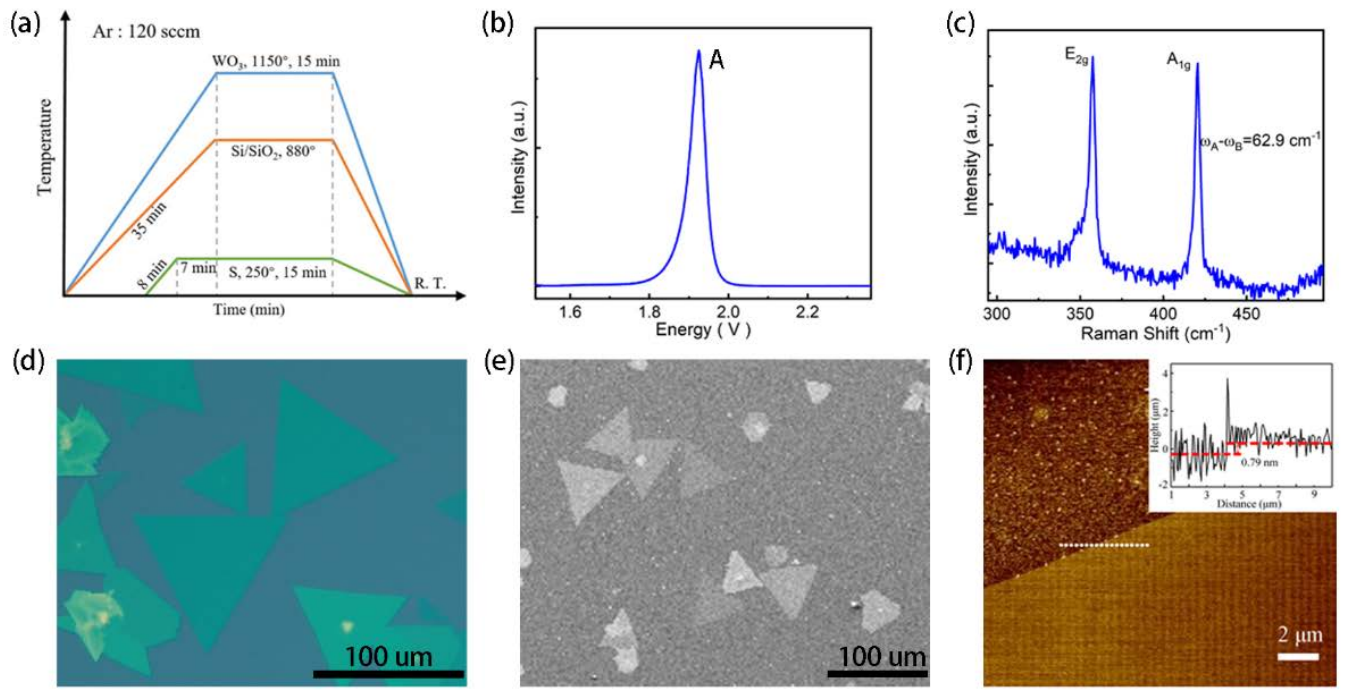

Figure S1. (a) Schematic growth process of $\mathrm{WS}_{2}$. Temperature, growth time and more detailed information are displayed. (b) PL spectrum acquired with $488 \mathrm{~nm}$ laser show a typical peak around $1.90 \mathrm{eV}$ of as grown monolayer $\mathrm{WS}_{2}$, implying a uniform flake. (c) Raman spectrum with two prominent peaks of as grown monolayer $\mathrm{WS}_{2}$, assigned to be $\mathrm{E}_{2 \mathrm{~g}}$ and $\mathrm{A}_{1 \mathrm{~g}}$ phonon mode, which located at $357.6 \mathrm{~cm}^{-1}$ and $420.5 \mathrm{~cm}^{-1}$ respectively. (d) Optical images showing monolayer WS 2 flakes on $\mathrm{SiO}_{2} / \mathrm{Si}$ substrate. Most flakes are triangular, but few flakes are irregular shape. (e) SEM image of monolayer $\mathrm{WS}_{2}$, demonstrating the good quality of crystallinity. (f) AFM image with a height profile (inset image), showing a step height of $0.79 \mathrm{~nm}$, which is a typical step height of monolayer $\mathrm{WS}_{2}$.

(a)

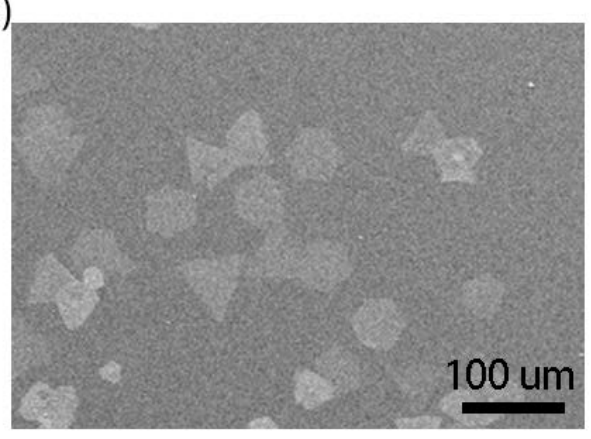

(b)

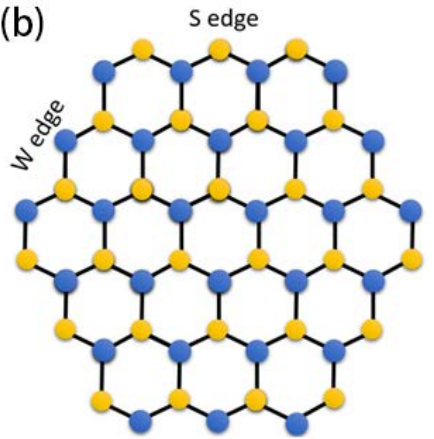

Figure S2. (a) SEM image of monolayer $\mathrm{WS}_{2}$ with triangular and hexagon shapes. (b) Schematic diagram of hexagonal $\mathrm{WS}_{2}$ flakes. 


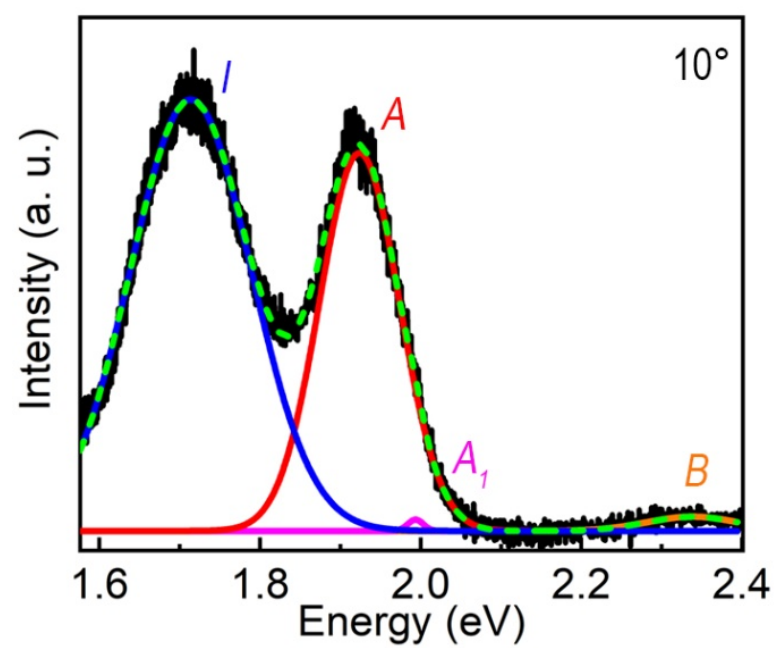

Figure S3. PL spectrum of $10^{\circ}$ twisted bilayer $\mathrm{WS}_{2}$. The green dashed curve is the sum of four Gaussian peaks with the color of blue, red, pink and orange respectively.

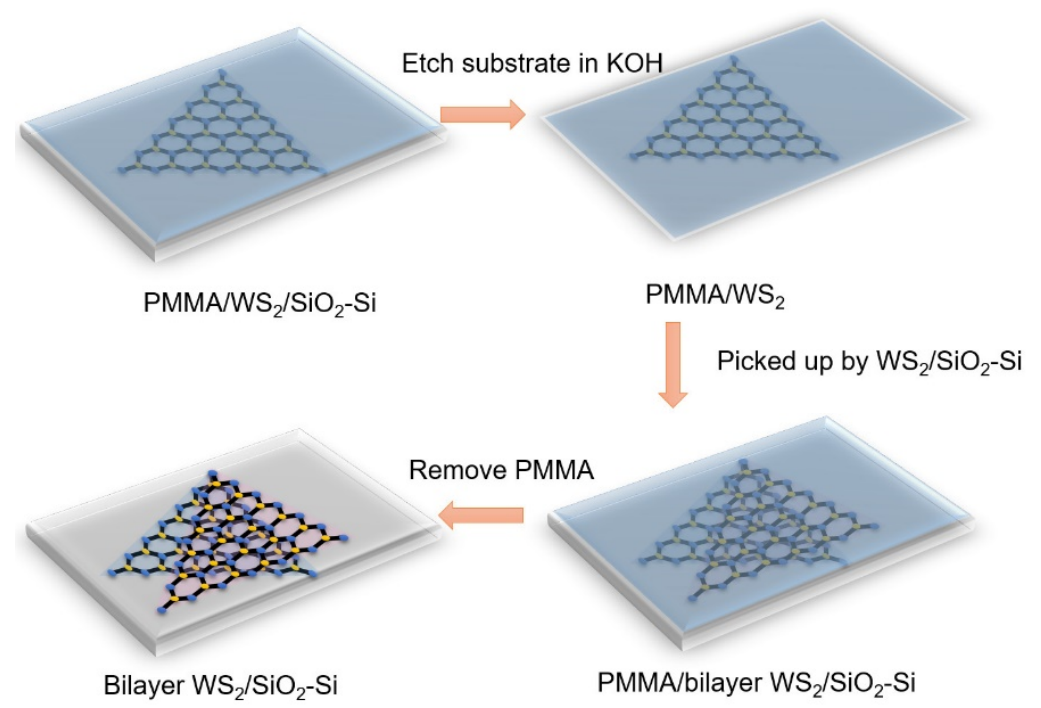

Figure S4. Schematic process of fabricating bilayer $\mathrm{WS}_{2}$ sample. (a) Spin coat a thin layer of PMMA on as-grown $\mathrm{WS}_{2}$ sample. (b) Etch $\mathrm{Si} / \mathrm{SiO}_{2}$ substrate using $20 \%$ potassium hydroxide $(\mathrm{KOH})$. (c) Stamp the PMMA/WS 2 monolayer onto a $\mathrm{SiO}_{2} / \mathrm{Si}$ substrate grown with $\mathrm{WS}_{2}$ monolayers in advance. (d) PMMA is removed in acetone steam. The bilayer samples were successfully prepared. 

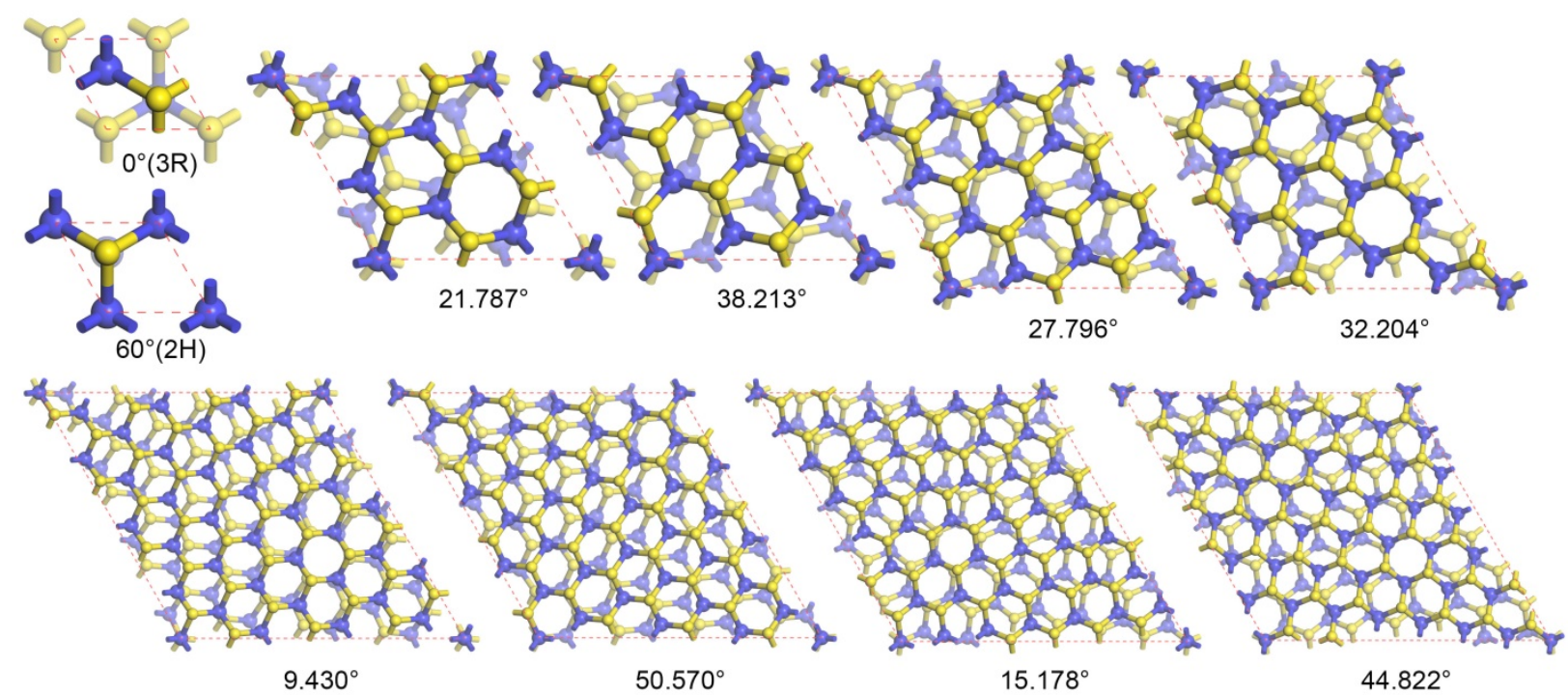

Figure S5. The optimized bilayer $\mathrm{WS}_{2}$ structures obtained by DFT calculations. The yellow and blue spheres represent $\mathrm{S}$ and $\mathrm{W}$ atoms respectively.
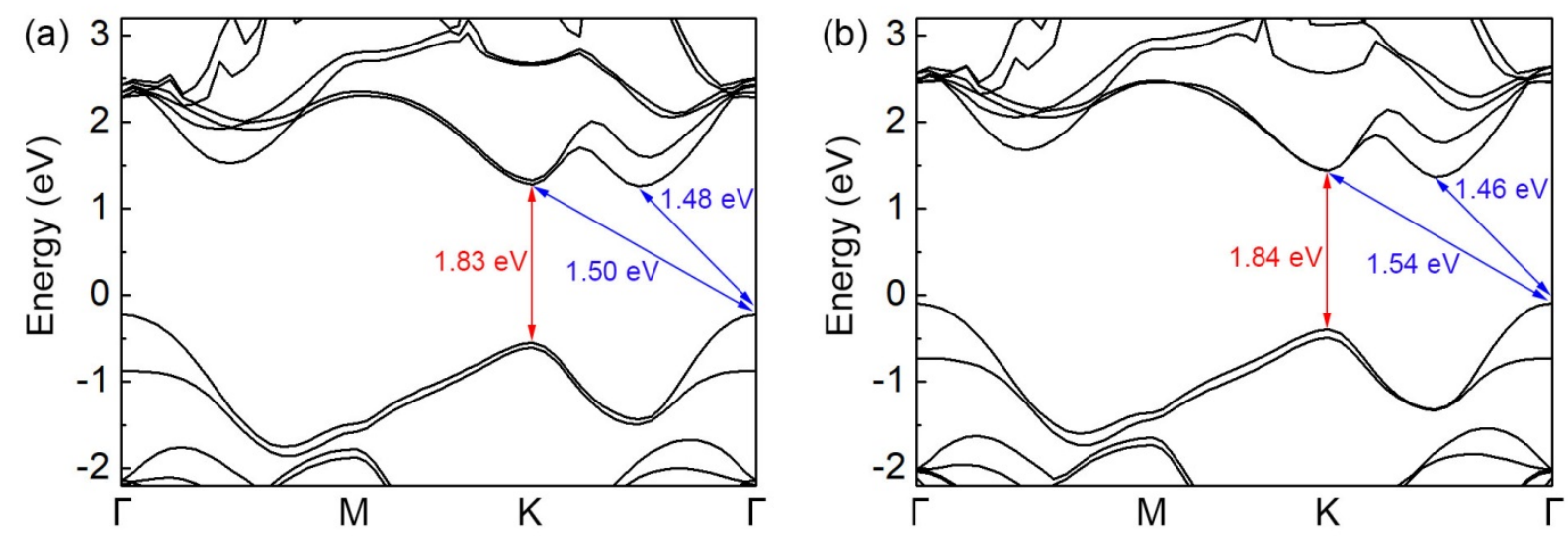

Figure S6. The band structure of (a) $0^{\circ}$ and (b) $60^{\circ}$ twisted bilayer $\mathrm{WS}_{2}$. Note that we adopt the dipole-allowed direct bandgap for bilayer $\mathrm{WS}_{2}$ with twist angles of $9.430^{\circ} / 50.570^{\circ}$ and $15.178^{\circ} / 44.822^{\circ}$. 

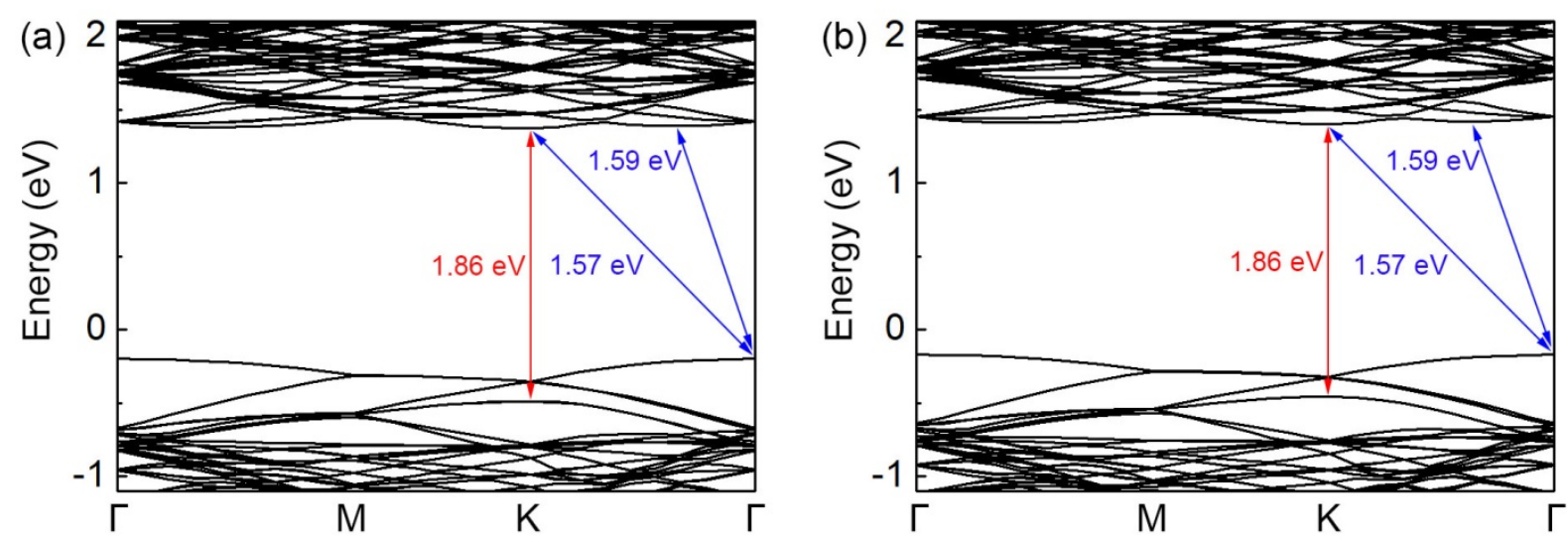

Figure S7. The band structure of (a) $9.430^{\circ}$ and (b) $50.570^{\circ}$ twisted bilayer WS 2 . Note that we adopt the dipole-allowed direct bandgap.
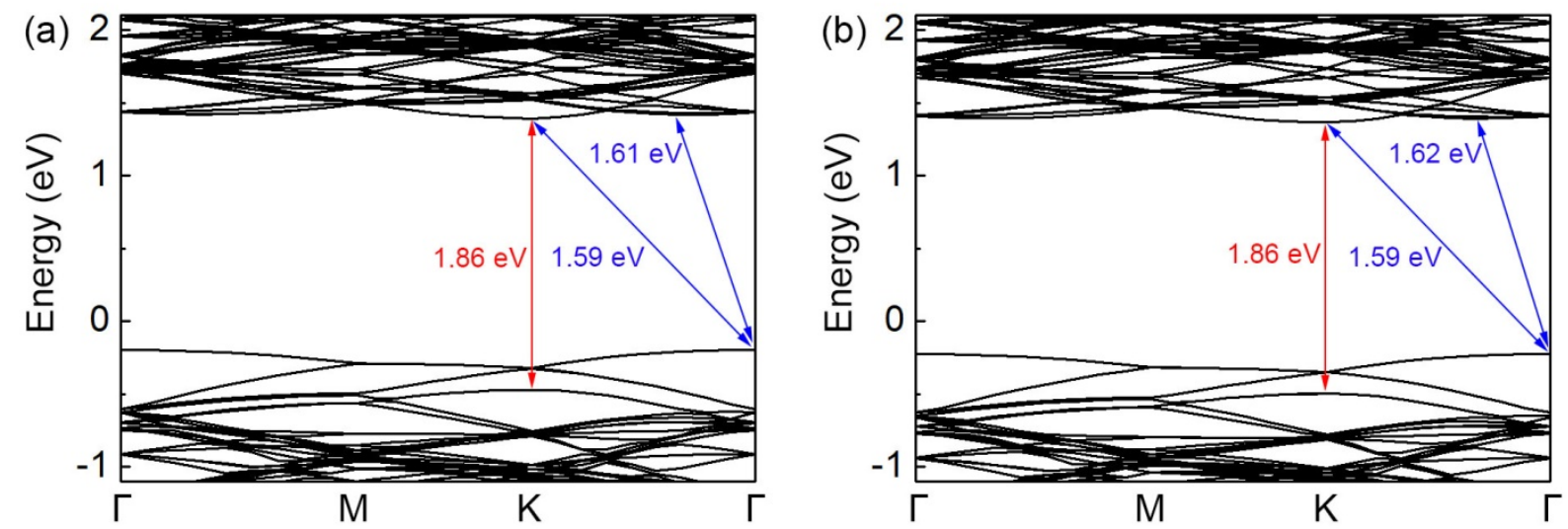

Figure S8. The band structure of (a) $15.178^{\circ}$ and (b) $44.822^{\circ}$ twisted bilayer $\mathrm{WS}_{2}$. Note that we adopt the dipole-allowed direct bandgap.
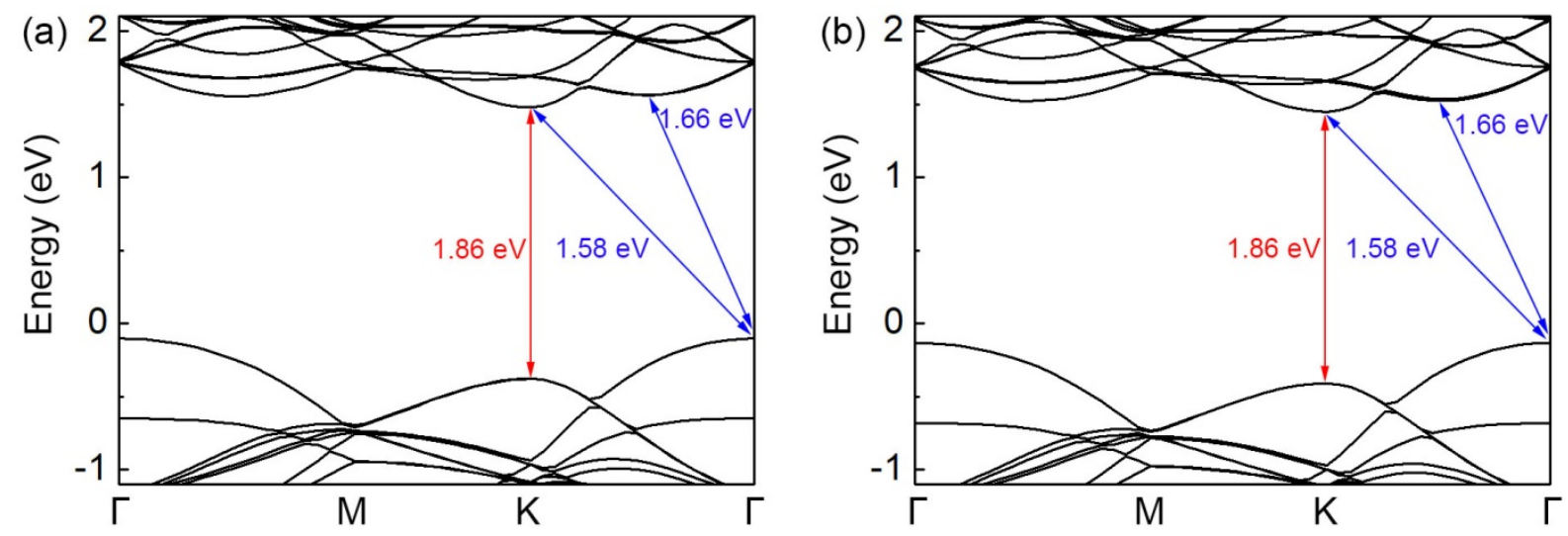
Figure S9. The band structure of (a) $21.787^{\circ}$ and (b) $38.213^{\circ}$ twisted bilayer $\mathrm{WS}_{2}$.
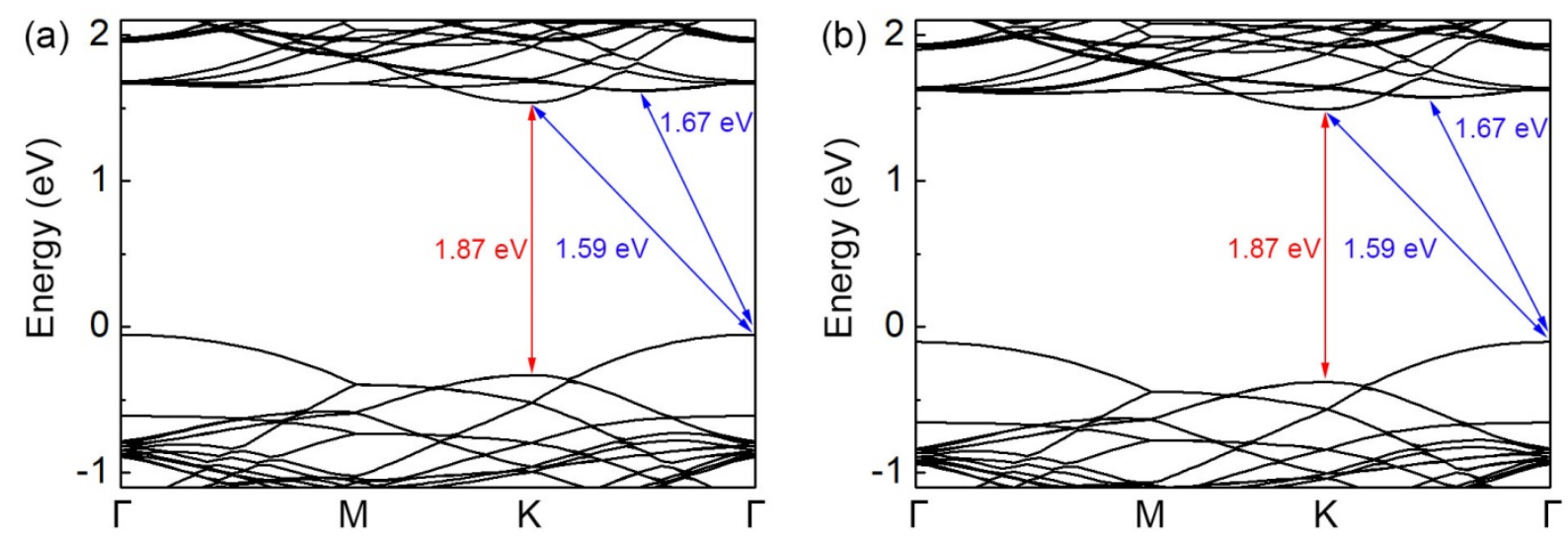

Figure S10. The band structure of (a) $27.796^{\circ}$ and (b) $32.204^{\circ}$ twisted bilayer $\mathrm{WS}_{2}$. 